\title{
Perfect powers in products of integers from a block of consecutive integers (II)
}

\author{
by
}

T. N. Shorey (Bombay) and Yu. V. Nesterenko (Moscow)

1. Introduction. For an integer $\nu>1$, we define $P(\nu)$ to be the greatest prime factor of $\nu$ and we write $P(1)=1$. Let $m \geq 0$ and $k \geq 2$ be integers. Let $d_{1}, \ldots, d_{t}$ with $t \geq 2$ be distinct integers in the interval $[1, k]$ and let $l>2, y>0$ and $b>0$ be integers with $P(b) \leq k$. We consider the equation

$$
\left(m+d_{1}\right) \ldots\left(m+d_{t}\right)=b y^{l}
$$

in $m, t, d_{1}, \ldots, d_{t}, b, y$ and $l$. We always assume that the left hand side of equation (1) is divisible by a prime exceeding $k$. Consequently, there is an $i$ with $1 \leq i \leq t$ such that $m+d_{i}$ is divisible by an $l$ th power of a prime exceeding $k$. Thus $m+d_{i} \geq(k+1)^{l}$ implying that $m>k^{l}$.

Equation (1) with $t=k$ and $b=1$ is solved completely by Erdös and Selfridge [5] in 1975; a product of two or more consecutive positive integers is never a power. In fact, Erdős [4] proved in 1955 that for $\varepsilon>0$, equation (1) with $b=1$ and

$$
t \geq k-(1-\varepsilon) k \frac{\log \log k}{\log k}
$$

implies that $k$ is bounded by an effectively computable number depending only on $\varepsilon$. This was sharpened considerably by Shorey [7], [8] in 1986-87. Shorey [8] showed that equation (1) with

$$
t \geq \frac{1}{2}\left(1+\frac{4 l^{2}-8 l+7}{2(l-1)\left(2 l^{2}-5 l+4\right)}\right) k
$$

implies that $k$ is bounded by an effectively computable absolute constant. Further, the assumption (2) has been relaxed for sufficiently large $l$. More precisely, Shorey [7] showed in 1986 that equation (1) with

$$
t \geq k l^{-1 / 11}+\pi(k)+2
$$

implies that $\min (k, l)$ is bounded by an effectively computable absolute constant. 
The proofs of these results depend on the method of Roth and Halberstam on difference between consecutive $\nu$-free integers, the results of Baker [1] on the approximations of algebraic numbers of the form $(A / B)^{m / n}$ with $A>B$ by rationals and the theory of linear forms in logarithms. The precise dependence on " $A$ " in the irrationality measures of Baker [1] plays a crucial role in the proofs. Further, Baker's sharpening [3] on linear forms in logarithms is essential. Linear forms in logarithms with $\alpha_{i}$ 's very close to 1 appear in the proofs and the best possible estimates of Shorey [7, Lemma 2], namely replacing $\log A$ in place of $\log A_{1} \ldots \log A_{n}$ with $A=\max _{1 \leq i \leq n} A_{i}$, for these linear forms in logarithms are required.

In this paper, we improve the results mentioned above on equation (1) whenever $l \geq 7$. For this, it is important to relax the assumption (2) of Baker [1] even though this makes the exponent of irrationality measure less precise. This is possible by appealing to a subsequent paper of Baker [2] in this direction. See Lemma 1. We shall also use an improved version, due to Loxton, Mignotte, van der Poorten and Waldschmidt [6], of Shorey [7, Lemma 2] cited above on linear forms in logarithms to relax the assumption (3). For stating the results of this paper, we define for $l \geq 7$,

$$
\nu_{l}= \begin{cases}\frac{112 l^{2}-160 l+29}{28 l^{3}-76 l+29} & \text { if } l \equiv 1(\bmod 2), \\ \frac{112 l^{2}-160 l+17}{28 l^{3}-188 l+129} & \text { if } l \equiv 0(\bmod 2) .\end{cases}
$$

For $l \geq 7$, we observe that $\nu_{l} \geq 3 / l$,

$$
\nu_{l} \leq \begin{cases}\frac{4}{l}\left(1-\frac{1}{(.875) l}\right) & \text { if } l \equiv 1(\bmod 2), \\ \frac{4}{l}\left(1-\frac{1}{(1.412) l}\right) & \text { if } l \equiv 0(\bmod 2)\end{cases}
$$

and

$$
\begin{aligned}
\nu_{7} & \leq .4832, \quad \nu_{8} \leq .4556, \quad \nu_{9} \leq .3878, & \nu_{10} \leq .3664, \\
\nu_{11} \leq .3243, & \nu_{12} \leq .3076, \quad \nu_{13} \leq .2787, & \nu_{14} \leq .2655 .
\end{aligned}
$$

We prove the following result.

ThEOrEm. (a) Equation (1) with

$$
l \geq 7, \quad t \geq \nu_{l} k
$$

implies that $k$ is bounded by an effectively computable number depending only on $l$.

(b) Let $\varepsilon>0$. There exists an effectively computable number $C$ depending only on $\varepsilon$ such that equation (1) with

$$
t \geq k l^{-1 / 3+\varepsilon}+\pi(k)+2
$$

implies that $\min (k, l) \leq C$. 
2. A relaxation in the assumption (2) of Baker's paper [1]. In this section, we appeal to Baker's paper [2] in order to derive the following result.

Lemma 1. Let $A, B, K$ and $n$ be positive integers such that $A>B$, $K<n, n \geq 3$ and $\omega=(B / A)^{1 / n}$ is not a rational number. For $0<\phi<1$, put

$$
\begin{aligned}
\delta=1+\frac{2-\phi}{K}, \quad s & =\frac{\delta}{1-\phi}, \\
u_{1}=40^{n(K+1)(s+1) /(K s-1)}, \quad u_{2}^{-1} & =K 2^{K+s+1} 40^{n(K+1)} .
\end{aligned}
$$

Assume that

$$
A(A-B)^{-\delta} u_{1}^{-1}>1
$$

Then

for all integers $p$ and $q$ with $q>0$.

$$
\left|\omega-\frac{p}{q}\right|>\frac{u_{2}}{A q^{K(s+1)}}
$$

Proof. We put

$$
\lambda_{1}=40^{n(K+1)} A, \quad \lambda_{2}=40^{n(K+1)}(A-B)^{K+1} A^{-K}
$$

and

$$
\Lambda=\frac{\log \lambda_{1}}{\log \lambda_{2}} .
$$

By (6) and $0<\phi<1$, we observe that $0<\lambda_{2}<1$. We follow Baker [2] with $m_{j}=j / n$ for $0 \leq j \leq K$ to conclude that for integers $r, p$ and $q$ with $r>0$ and $q>0$, there exists a polynomial $P_{r}(X) \in \mathbb{Z}[X]$ satisfying
(i) $\operatorname{deg} P_{r} \leq K$,
(ii) $H\left(P_{r}\right) \leq \lambda_{1}^{r}$,
(iii) $P_{r}(p / q) \neq 0$,
(iv) $\left|P_{r}(w)\right| \leq \lambda_{2}^{r}$.

Here $H\left(P_{r}\right)$ denotes the maximum of the absolute values of the coefficients of $P_{r}$. For $r \geq 54$, Baker [2] gave sharper estimates (ii) and (iv) with 40 replaced by 4 in the definitions (7) of $\lambda_{1}$ and $\lambda_{2}$. We may assume that $|\omega-p / q|<1 / 2$ and we define $r$ as the smallest integer such that

$$
\lambda_{2}^{r} \leq \frac{1}{2 q^{K}} .
$$

Then

and

$$
\lambda_{2}^{r}>\frac{\lambda_{2}}{2 q^{K}}
$$

$$
\lambda_{1}^{r}=\left(\lambda_{2}^{r}\right)^{\Lambda} \leq\left(\frac{\lambda_{2}}{2 q^{K}}\right)^{\Lambda}=\lambda_{1} 2^{-\Lambda} q^{-K \Lambda} .
$$


Further, we observe that

$\frac{1}{q^{K}} \leq\left|P_{r}\left(\frac{p}{q}\right)\right| \leq\left|P_{r}\left(\frac{p}{q}\right)-P_{r}(\omega)\right|+\left|P_{r}(\omega)\right| \leq\left|P_{r}\left(\frac{p}{q}\right)-P_{r}(\omega)\right|+\frac{1}{2 q^{K}}$.

Thus

On the other hand, we have

$$
\left|P_{r}\left(\frac{p}{q}\right)-P_{r}(\omega)\right| \geq \frac{1}{2 q^{K}} .
$$

$$
\left|P_{r}\left(\frac{p}{q}\right)-P_{r}(\omega)\right|=\left|\int_{p / q}^{\omega} P_{r}^{\prime}(X) d X\right| \leq K 2^{K} \lambda_{1}^{r}\left|\omega-\frac{p}{q}\right| .
$$

Consequently,

$$
\left|\omega-\frac{p}{q}\right|>\left(K 2^{K+1} \lambda_{1}\right)^{-1} 2^{\Lambda} q^{-\chi}
$$

where $\chi=K-K \Lambda$. By (6), we observe that $-\Lambda \leq s$ and $\chi \leq K(s+1)$. Hence

$$
\left|\omega-\frac{p}{q}\right|>\frac{u_{2}}{A q^{K(s+1)}} .
$$

3. Proof of Theorem (a). Let $\varepsilon_{1}=\left(10^{6} l^{3}\right)^{-1}$. Suppose that equation (1) with (4) is satisfied. We may assume that $k$ exceeds a sufficiently large effectively computable number depending only on $l$. We denote by $u_{3}, u_{4}$ and $u_{5}$ effectively computable positive numbers depending only on $l$. We put

$$
\tau=\left(1+\frac{\varepsilon_{1} l}{4}\right) \nu_{l}^{-1}<\frac{l}{2}, \quad \tau_{1}=\left(\nu_{l}^{-1}-1\right) /(l-1) .
$$

We see from equation (1) that

$$
m+d_{i}=a_{i} x_{i}^{l} \quad \text { for } 1 \leq i \leq t,
$$

where $a_{i}$ and $x_{i}$ are positive integers satisfying

$$
P\left(a_{i}\right) \leq k, \quad\left(x_{i}, \prod_{p \leq k} p\right)=1 .
$$

We write $S=\left\{a_{1}, \ldots, a_{t}\right\}$. We argue as in [8] to conclude that there exists a subset $S_{2}$ of $S$ with $\left|S_{2}\right| \geq u_{3} k$ and

$$
a_{i} \leq k^{\tau} \quad \text { for } a_{i} \in S_{2} .
$$

Further we apply the method of Halberstam and Roth as in [8] for deriving that there exists a subset $S_{3}$ of $S_{2}$ with $\left|S_{3}\right| \geq u_{4} k^{1-\varepsilon_{1}}$ such that

$$
x_{i}>k^{2-\tau_{1}-5 \varepsilon_{1}} \quad \text { for } a_{i} \in S_{3} .
$$


In fact, (9) is valid with $\tau$ replaced by $\tau^{\prime}=\left(1+\varepsilon^{\prime} / 4\right) \nu_{l}^{-1}$ where $\varepsilon^{\prime}=$ $\left(10^{6} l^{5}\right)^{-1}$, and we use this estimate for deriving (10). Put $s_{3}=\left|S_{3}\right|$. By permuting the subscripts of $d_{1}, \ldots, d_{t}$, there is no loss of generality in assuming that $a_{1}, a_{2}, \ldots, a_{s_{3}}$ are elements of $S_{3}$ and $a_{1}<a_{2}<\ldots<a_{s_{3}}$. Then we find, as in [8], an integer $\mu$ with $1 \leq \mu<s_{3}$ such that

$$
\log \left(\frac{a_{\mu+1}}{a_{\mu}}\right) \leq \frac{u_{5} \log k}{k^{1-\varepsilon_{1}}}
$$

and

$$
0 \neq\left|\left(\frac{a_{\mu}}{a_{\mu+1}}\right)^{1 / l}-\frac{x_{\mu+1}}{x_{\mu}}\right|<\frac{2 k}{a_{\mu+1} x_{\mu}^{l}} .
$$

Now, we turn to applying Lemma 1 with

$$
K= \begin{cases}(l-3) / 2 & \text { if } l \equiv 1(\bmod 2), \\ (l-4) / 2 & \text { if } l \equiv 0(\bmod 2),\end{cases}
$$

and $A=a_{\mu+1}, B=a_{\mu}, n=l$. We put $\psi=(2-\phi) / K$, where $\phi$ will be chosen later in some special way and we put $\delta=1+\psi$ with $2 /(l-3)<\psi<1$. By (11), we observe that

$$
\frac{a_{\mu+1}-a_{\mu}}{a_{\mu+1}}<\frac{a_{\mu+1}-a_{\mu}}{a_{\mu}}<\frac{2 u_{5} \log k}{k^{1-\varepsilon_{1}}} .
$$

Therefore, by (9), the left hand side of inequality (6) exceeds

$$
\left(\frac{k^{1-\varepsilon_{1}}}{2 u_{5} \log k}\right)^{1+\psi}\left(u_{1} k^{\tau \psi}\right)^{-1} \text {. }
$$

Thus, the assumption (6) is satisfied if $1+\psi-\tau \psi \geq 5 \varepsilon_{1}$, which, by (8), reads

$$
\nu_{l} \geq \frac{\psi}{1+\psi}+\frac{\varepsilon_{1} l}{4} \cdot \frac{\psi}{1+\psi}+\frac{5 \varepsilon_{1} \nu_{l} \psi}{1+\psi} .
$$

We observe that the second summand on the right hand side of the preceding inequality does not exceed $2 \varepsilon_{1}$, since

$$
\frac{\psi}{1+\psi}=\frac{2-\phi}{K+2-\phi}<\frac{2}{K+1} \leq \frac{4}{l-2},
$$

and the third summand is at most $5 \varepsilon_{1}$, since $\nu_{l}<1$ and $0<\psi<1$. Hence, the assumption (6) is satisfied if

$$
\nu_{l} \geq \frac{\psi}{1+\psi}+7 \varepsilon_{1} .
$$

We shall later choose $\phi$ depending only on $l$ so that (14) is satisfied. Then, the assumption of Lemma 1 is valid. Hence, we conclude from Lemma 1 that

$$
\left|\left(\frac{a_{\mu}}{a_{\mu+1}}\right)^{1 / l}-\frac{x_{\mu+1}}{x_{\mu}}\right|>\frac{u_{2}}{a_{\mu+1} x_{\mu}^{K(s+1)}} .
$$


We put $\theta=l-K(s+1)$. The parameter $\phi$ will be chosen later in such a way that $\theta>0$. We observe from (5) that

$$
\theta=l-\frac{K+2-\phi}{1-\phi}-K=l-\left(2+\frac{\phi}{1-\phi}\right)(K+1)
$$

which, by (13), implies that

$$
\theta=\theta^{\prime}-\frac{\phi(K+1)}{1-\phi}
$$

where

$$
\theta^{\prime}= \begin{cases}1 & \text { if } l \equiv 1(\bmod 2) \\ 2 & \text { if } l \equiv 0(\bmod 2)\end{cases}
$$

Further, we see from (8) and (14) that

$$
\tau_{1} \leq \frac{1}{(l-1) \psi}-\varepsilon_{1} .
$$

Finally, we combine (12), (15) and (10) in order to derive that

$$
k^{\left(2-\tau_{1}-5 \varepsilon_{1}\right) \theta}<2 u_{2}^{-1} k,
$$

which, since $k$ is sufficiently large, implies that $\left(2-\tau_{1}-5 \varepsilon_{1}\right) \theta<1+\varepsilon_{1}$. Consequently,

$$
\theta^{\prime}-\frac{\phi(K+1)}{1-\phi}<\left(2-\frac{1}{(l-1) \psi}\right)^{-1}+8 \varepsilon_{1}
$$

Let $l \equiv 1(\bmod 2)$. Then, by substituting $\theta=1, l=2 K+3$ and $\psi=$ $(2-\phi) / K$, we get

$$
(1-(K+2) \phi)(7 K+8-(4 K+4) \phi)-(2 K+2)\left(2-3 \phi+\phi^{2}\right)<128 \varepsilon_{1} K .
$$

Thus

$$
\left(4 K^{2}+10 K+6\right) \phi^{2}-\left(7 K^{2}+20 K+14\right) \phi+3 K+4<128 \varepsilon_{1} K .
$$

Let

$$
\phi=\frac{24 K+28.84}{14\left(4 K^{2}+10 K+6\right)} .
$$

Then

$$
(45.68) K^{2}-(26.88) K-116.8944<3 \cdot 10^{6} \varepsilon_{1} K^{3} .
$$

We observe that the left hand side of the preceding inequality exceeds 12 since $K \geq 2$. On the other hand, the right hand side is less than one. This is a contradiction. 
Let $l \equiv 0(\bmod 2)$. Then

$$
\left(4 K^{2}+16 K+15\right) \phi^{2}-\left(7 K^{2}+35 K+39\right) \phi+10 K+18<128 \varepsilon_{1} K
$$

and we choose

$$
\phi=\frac{80 K+127.82}{14\left(4 K^{2}+16 K+15\right)}
$$

to obtain

$$
(145.64) K^{2}-(12.6) K-531.7676<3 \cdot 10^{6} \varepsilon_{1} K^{3},
$$

leading to a contradiction. Finally, we compute $\psi$ in either of the cases $l \equiv 1$ $(\bmod 2)$ and $l \equiv 0(\bmod 2)$ to observe that the assumption $(14)$ is valid. This completes the proof of Theorem (a).

4. Proof of Theorem (b). We follow the notation of [7, Lemma 2] where, under certain assumptions, the lower bound

$$
\exp \left(-\left(C_{9} \tau_{2} n^{3}\right)^{3 n+3} \tau_{1} \log A\right)
$$

for the absolute value of linear forms in logarithms was proved. This has been improved to

$$
\exp \left(-\left(C_{9} n\right)^{n} \tau_{2}^{n+1} \log A\right)
$$

in [6, Theorem 1]. If we replace (16) by (17) for the case $n=2$ in the proof of [7, Lemma 6], the assertion of Theorem (b) follows.

\section{References}

[1] A. Baker, Rational approximations to $\sqrt[3]{2}$ and other algebraic numbers, Quart. J. Math. Oxford Ser. (2) 15 (1964), 375-383.

[2] - Simultaneous rational approximations to certain algebraic numbers, Proc. Cambridge Philos. Soc. 63 (1967), 693-702.

[3] - The theory of linear forms in logarithms, in: Transcendence Theory: Advances and Applications, Academic Press, 1977, 1-27.

[4] P. Erdős, On the product of consecutive integers III, Indag. Math. 17 (1955), 85-90.

[5] P. Erdős and J. L. Selfridge, The product of consecutive integers is never a power, Illinois J. Math. 19 (1975), 292-301.

[6] J. H. Loxton, M. Mignotte, A. J. van der Poorten and M.Waldschmidt, A lower bound for linear forms in the logarithms of algebraic numbers, C. R. Math. Rep. Acad. Sci. Canada 11 (1987), 119-124.

[7] T. N. Shorey, Perfect powers in values of certain polynomials at integer points, Math. Proc. Cambridge Philos. Soc. 99 (1986), 195-207. 
[8] T. N. Shorey, Perfect powers in products of integers from a block of consecutive integers, Acta Arith. 49 (1987), 71-79.

School of Mathematics

Tata Institute of Fundamental Research

Homi Bhabha Road

Bombay 400 005, India

E-mail: shorey@math.tifr.res.in
Department of Mathematics University of Moscow

Moscow 119899, Russia

E-mail: nest@nw.math.msu.su

Received on 30.6.1995

and in revised form on 29.12.1995 